Editor's Note: Toolboxes are a new, occasional feature in the Journal designed to briefly highlight a new method or a resource of general use in neuroscience or to critically analyze existing approaches or methods. For more information, see http://www. jneurosci.org/misc/ifa_features.shtml.

\title{
Antibodies as Valuable Neuroscience Research Tools versus Reagents of Mass Distraction
}

\author{
Kenneth J. Rhodes ${ }^{1}$ and James S. Trimmer ${ }^{2}$ \\ ${ }^{1}$ CNS Research, Johnson and Johnson Pharmaceutical Research and Development, Spring House, Pennsylvania 19477, and ${ }^{2}$ Department of Pharmacology, \\ School of Medicine, University of California, Davis, California 95616
}

Antibody-based approaches for isolation, characterization and localization of target proteins are among the most critical and widely used techniques in molecular and cellular neuroscience. In many cases, the antibodies used for such studies are products of painstaking efforts by academic and industrial neuroscientists to generate high-quality, well characterized reagents for their own research. Through generosity, collaboration, or commercial licensing, many investigators have made their antibodies available to other neuroscientists, leading to rapid expansion in our knowledge of protein abundance, distribution, structure, and function. In a perfect world, antibodies would be generated and validated in an iterative process that includes rigorous evaluation at independent laboratories followed by dissemination of relevant information throughout the research community. As a result, limitations of a particular antibody reagent would be widely recognized and stimulate efforts to generate new antibodies with improved activity or specificity. The end

Received June 27, 2006; revised June 28, 2006; accepted June 29, 2006.

Dr. Rhodes is an advisory board member and Dr. Trimmer is the Director of NeuroMab. NeuroMab is supported by National Institute of Neurological Disorders and Stroke/National Institute of Mental Health Grant U24NS50606. We thank Dr. Milena Menegola for images and helpful comments, Dr. Randy Stewart for helpful comments, and past and current colleagues for their invaluable contributions to antibody generation and characterization in our respective laboratories.

Correspondence should be addressed to Dr. James S. Trimmer, Department of Pharmacology, School of Medicine, 3502 GBSF, University of California, One Shields Avenue, Davis, CA 95616. E-mail: jtrimmer@ucdavis.edu.

DOI:10.1523/JNEUROSCI.2728-06.2006

Copyright $\odot$ 2006 Society for Neuroscience $\quad 0270-6474 / 06 / 268017-04 \$ 15.00 / 0$ result would be global availability of well characterized antibodies that can be reliably used to study a range of properties of specific target proteins. Such well characterized and validated antibodies have tremendous value to the research community because they yield reproducible results across independent laboratories, leading to significant advances in the field. However, it is not a perfect world. Often antibodies lack the requisite efficacy and/or specificity; thus, investigators can suffer undue diversion of research effort and funds, and in a worse case scenario, contribute to mass distraction by initiating or perpetuating erroneous findings.

The success of the Human Genome Project has placed a renewed emphasis on proteomic approaches in neuroscience. New methods for immunogen production, animal immunization, and antibody generation and validation have matured such that it is now possible to generate antibodies against a wide range of target proteins, regardless of their biochemical characteristics or natural abundance. Moreover, commercial antibody companies have proliferated and offer for sale antibodies directed at a wide range of proteins, freeing individual investigators to focus on application of antibody-based applications and not the generation and validation process itself. For some protein classes, commercially generated antibodies represent the primary source of antibodies used in academic and industrial research. Unfortunately, in many cases commercial antibodies fail even the most fundamental tests of activity and/or specificity (e.g., selective staining of cells overexpressing the target protein versus those that do not express the target protein at all), and with alarming regularity vendors pass the burden for antibody validation/ quality control to the end user. The end user can decide to either simply use the antibody as is for her/his intended research goals, with the assumption that the vendor has performed adequate quality control to demonstrate activity and specificity, or become distracted from these goals and instead spend valuable research funds and effort to critically evaluate the antibody before use and eventual publication of their results. In some fields of neuroscience, the poor quality of antibody reagents has caused considerable frustration among investigators and led to publication and perpetuation of erroneous research results.

Recognizing the highly variable quality and level of characterization of antibodies generated in both academic and commercial laboratories for immunohistochemical staining, and in response to publication of results later found to be invalid because of the employment of poor quality antibodies, The Journal of Comparative Neurology (JCN) has implemented strict measures to ensure that manuscripts reporting antibody-based studies include detailed descriptions of the methods used to validate specificity of all antibodies used (Saper and Sawchenko, 2003). Although the requirement of the JCN to use limited research resources (including 
stocks of antibodies) in assays aimed at simply validating specificity of commercially obtained, expensive research reagents may seem extreme and draconian to some, journal editors must strive to ensure publication of results that will withstand scrutiny to avoid propagation of erroneous findings. This is a laudable goal, but one that may be out of reach of individual investigators not experienced or expert in antibody generation and characterization, or with funding or personnel limitations that do not allow for the purchase of expensive reagents and their extensive validation. The questions before us, then, are as follows: how can an investigator who may have little or no experience in antibody characterization use an antibody to answer a specific research question and move on, confident that the results obtained are accurate? What level of antibody characterization should an investigator perform in her/his own laboratory before results should be submitted for publication? What is the appropriate forum for providing critical feedback to commercial antibody companies (and academic laboratories) to encourage them to more thoroughly characterize and validate the antibodies that they sell for research use?

\section{Leveling the playing field}

Antibodies are affinity reagents. Although high-quality antibodies will exhibit a high affinity (low nanomolar $K_{\mathrm{D}}$ corresponding to $\approx 0.1-1 \mu \mathrm{g} / \mathrm{ml}$ for IgG), and therefore a high degree of efficacy and specificity for their target antigen, at high antibody concentrations or in complex protein mixtures (e.g., brain membrane extracts, cell lysates, or brain sections), even the best antibodies can show crossreactivity with "off-target" antigens. Although in some cases high-quality and reliable results can be obtained with crude polyclonal antisera, in other cases even the most highly purified polyclonal antibody (pAb) preparations (i.e., affinity-purified antibody preparations) may contain antibodies with reactivity against closely related antigenic sequences, and this crossreactivity contributes to or even dominates the observed signal. The techniques associated with monoclonal antibody $(\mathrm{mAb})$ production and purification offer the advantage that, unlike polyclonal antisera, in which the pAb of interest is a minor component of the total serum antibody pool, the mAb is the vastly predominant or sole antibody present in the material with which to begin purification. In applying the different types of antibod-
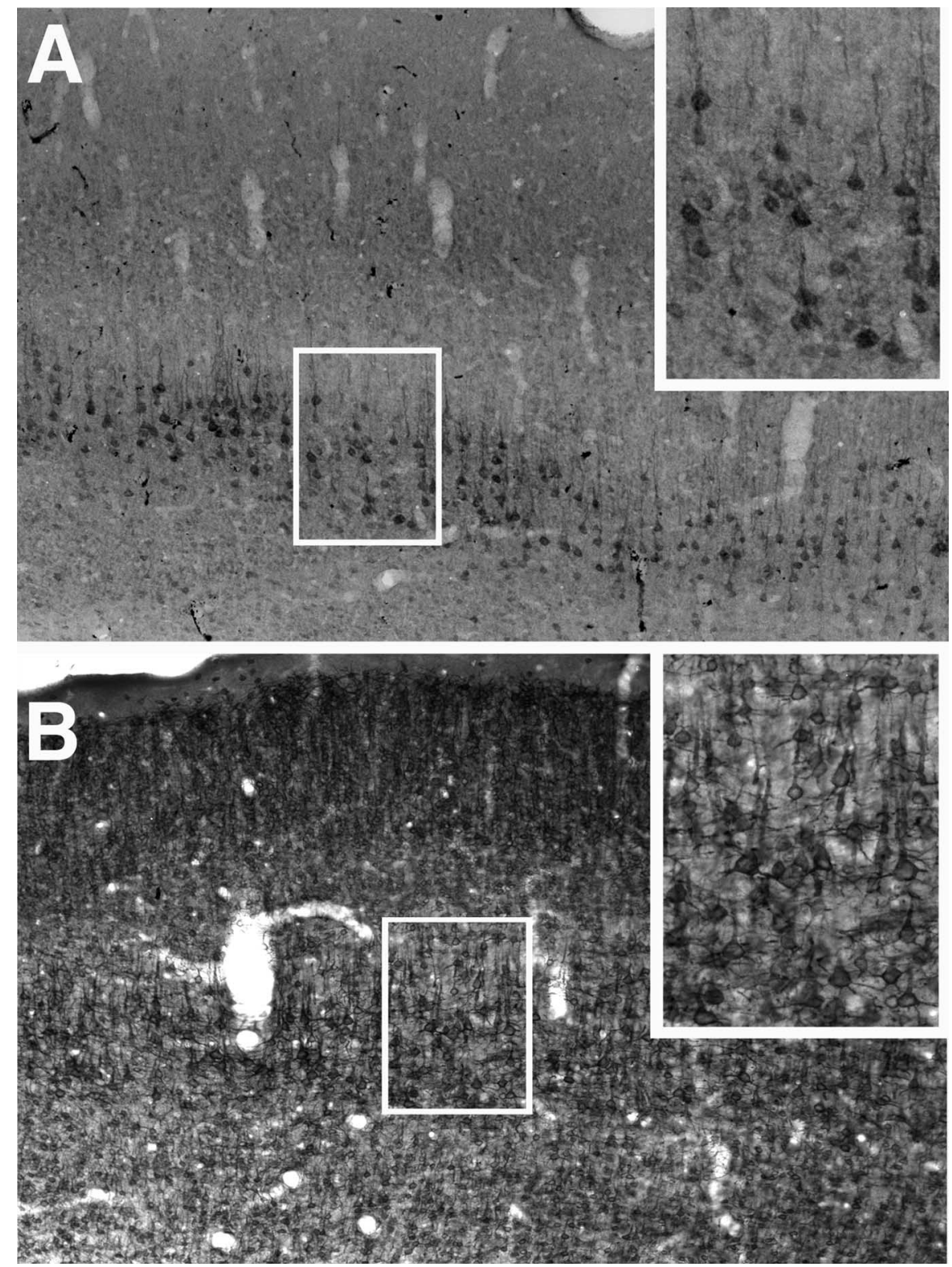

Figure 1. Examples of nonspecific and specific nickel-enhanced diaminobenzidine staining for somatodendritic ion channels from adult rat neocortex. A, A common nonspecific staining pattern. In this case, an ELISA-positive mAb against Kv3.1 yields cytoplasmic (not plasma membrane) staining in neocortical layer V pyramidal neurons, which do not express Kv3.1 mRNA (Weiser et al., 1994). B, Specific staining for Kv2.1. Note the crisp plasma membrane-associated staining, as expected for an ion channel, and little cytoplasmic or nuclear signal. Insets are higher-magnification views of the boxed area.

ies in cellular and molecular neuroscience, one has to recognize the potential for off-target binding and therefore carefully select the criteria and conditions under which the specificity of the antibody reagent is most likely (and adequately) demonstrated.

\section{Considerations specific}

\section{to immunohistochemistry}

The limitations of antibodies are most clearly exposed when these reagents are used for immunohistochemistry (IHC), especially on brain tissue sections. This is mainly because of the complex and heterogeneous cellular and molecular compo- sition of the brain relative to other tissues, and the fact that cues that are available to evaluate the specificity of the antibody signal in other experimental settings (e.g., reactivity against a single entity of appropriate molecular mass or electrophoretic mobility in immunoblotting) are not available for IHC. Reaction product or fluorescence reveals all sites at which the antibodies have bound, leaving considerable room for interpretation and confusion as to which staining is specific (i.e., represents the presence of the target antigen) and which is nonspecific (closely related or unrelated antigens). Therefore, 
when applying antibodies in IHC one has to look for convergence and concordance of data obtained using several methods to ensure that the interpretation of the observed staining pattern is appropriate.

One approach to obtain convergent data is to apply a combination of in situ hybridization (ISH) histochemistry and IHC techniques. By careful application of ISH histochemistry, or through careful analyses of ISH data, either in publications or in online atlases (e.g., the Allen Brain Atlas; www.brainatlas.org) one determines the brain regions and cell types that are expressing mRNA encoding the target antigen. The mRNA distribution provides a context to interpret the IHC staining pattern; if the IHC signal is localized to somata or dendrites, the ISH signal should be present in the same brain regions and cell types. If the IHC signal is localized to axonal processes, an investigator with knowledge of neuroanatomical connections should be able to determine whether the staining is in pathways or terminal fields that originate from cells expressing the corresponding mRNA. Such convergent data obtained from two independent methods provides an extremely powerful confirmation of the antibody signal (Rhodes et al., 1996). It is also critical to keep in mind the function of the target protein and its logical subcellular localization within the neuron. Plasma membrane channels and receptors should yield sharply defined plasma membrane and not diffuse cytoplasmic staining (Fig. 1 ), whereas transcription factors should exhibit nuclear staining, etc.

Another approach to obtaining concordant data is to compare the IHC staining patterns revealed by two different antibody preparations raised against distinct, nonoverlapping epitopes on the same target protein. Because it is highly unlikely that antibodies raised against nonoverlapping antigenic sequences from the same target antigen will show the same specific and nonspecific staining patters, areas of close overlap in IHC staining for the two antibodies are very likely to represent a specific signal. This approach to confirming mRNA localization is widely used for ISH because it is comparatively straightforward to generate multiple cRNA or cDNA probes directed at nonoverlapping segments of the target mRNA. Although it can be much more challenging, and rarely performed, investigators have generated high-quality antibodies to separate regions of the target protein. This approach is one of the most powerful to confirm specificity of the IHC
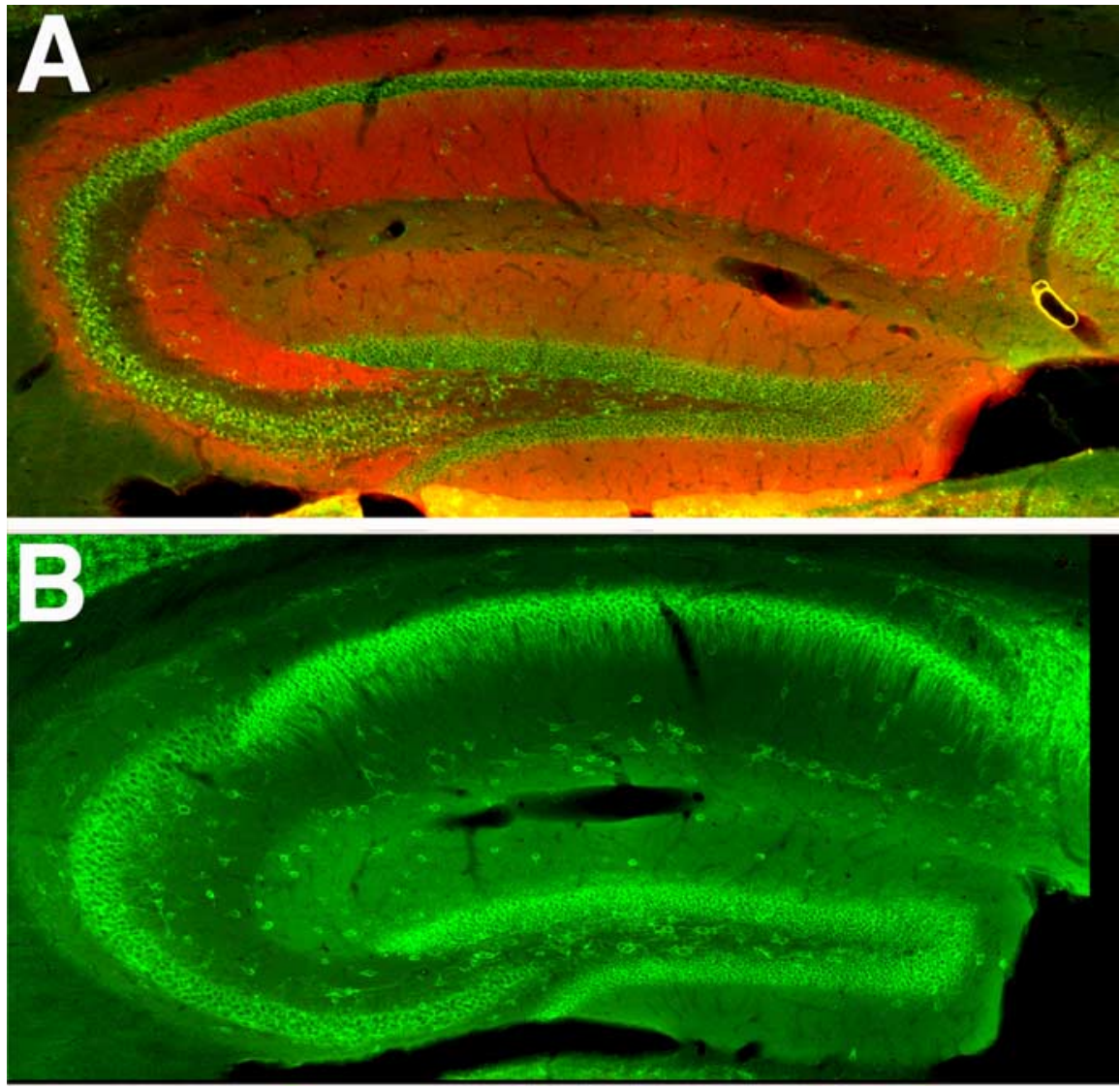

Figure 2. Validation of antibody staining for a dendritic ion channel using knock-out mice. Pattern of double immunofluorescence staining of an anti-Kv4.2 mAb (K57/1; red) and an anti-Kv2.1 pAb (green) against hippocampi from adult wild-type $(\boldsymbol{A})$ and Kv4.2 knock-out (B) mice.

signal. mAb projects can often yield multiple and independent mAb clones with distinct and nonoverlapping binding sites on the same target protein; convergence of staining patterns obtained with such a panel of mAbs can yield a strong confirmation of specificity.

\section{Monoclonal antibody screening as a general model for antibody validation} Obtaining good antibodies for IHC can be exceedingly simple or frustratingly difficult. There are numerous examples where crude polyclonal antiserum is of sufficient specificity to be used without additional purification for IHC, and of $\mathrm{mAb}$ projects wherein virtually every $\mathrm{mAb}$ obtained yields beautiful IHC. However, in many cases, obtaining pAbs or mAbs that work well for IHC requires extensive, multistep purification/adsorption steps (for pAbs), or analyses of large numbers of otherwise positive clones (for mAbs) to find even one that gives acceptable IHC staining. Over the years we have screened $>5000$ ELISA-positive mAbs (in $>100 \mathrm{mAb}$ projects) by IHC staining of mammalian brain sections. Typically, initial screens require a high-throughput format (e.g.,
ELISA) that is amenable to large numbers of samples $(\approx 1000$ for a typical $\mathrm{mAb}$ project). In addition to other screens (immunofluorescence staining of transfected cells, immunoblotting of brain preparations), we explicitly screen 50-100 ELISA-positive clones for IHC staining of mammalian brain sections (BekeleArcuri et al., 1996). It is not uncommon to find that from the ELISA-positive pool of $50-100$ clones, only a small number (1-5) yield high-quality IHC staining of brain sections, and that these do not typically correspond to the strongest ELISApositive clones. Based on this, we suggest that investigators who contract out $\mathrm{mAb}$ production with the intent of obtaining a reagent that can be used for IHC request and test as many ELISA-positive clones as is practical. Standard controls (omission of primary and secondary antibodies, immunogen competition, etc.), as elegantly described by Saper and Sawchenko (2003), should be included. Such analyses should be performed at all steps of the $\mathrm{mAb}$ evaluation (subcloning, large-scale production, purification), and similarly for $\mathrm{pAb}$ preparations (crude sera, IgG 
fractions, affinity-purified preparations, etc.). Additional evaluation by comparison with mRNA distribution, staining in sections from knock-out animals in which the target antigen has been genetically deleted (Fig. 2) or expression levels have been attenuated by siRNA, can and should be used, when available, to further verify the specificity of the observed staining patterns. For all antibody reagents, lack of IHC signal in tissues from knock-out animals in which the antigen has been eliminated is the best demonstration of antibody specificity.

We have recently implemented the approach detailed above to systematically generate mAbs for important neuroscience targets at the National Institutes of Health-funded University of California Davis/National Institute of Neurological Disorders and Stroke/National Institute of Mental Health NeuroMab facility. The goal is to generate high quality mAbs that are validated using the above procedures, with a specific focus on mammalian-brain immunoblotting and IHC, and to make these validated NeuroMabs available to the research community on a nonprofit basis. Although this single facility, whose goal is to generate NeuroMabs against 50 targets per year, cannot immediately address the acute antibody needs of the field of neuroscience, it may provide a model for parallel academic or industrial efforts aimed at generating high-quality antibodies for neuroscience research through careful, systematic validation procedures, including rigorous brain IHC, thus allowing end users to focus on experiments requiring their respective neurobiological expertise, as opposed to being distracted by spending critical time and effort on antibody validation/quality control that should be performed before, not after, distribution. As a way to better disseminate information as to the reliability of antibodies for neuroscience research, the NeuroMab website (www.neuromab. org) has initiated a message board for comments on end user experiences using NeuroMabs, and other widely available antibodies from nonprofit or commercial sources, for neuroscience applications. Hopefully, this will allow investigators to identify anti- bodies with proven track records in the neuroscience community, and avoid the distraction of wasted time and effort using antibodies that have a history of yielding poor results.

\section{References}

Bekele-Arcuri Z, Matos MF, Manganas L, Strassle BW, Monaghan MM, Rhodes KJ, Trimmer JS (1996) Generation and characterization of subtype-specific monoclonal antibodies to $\mathrm{K}^{+}$channel $\alpha$ - and $\beta$-subunit polypeptides. Neuropharmacology 35:851-865.

Rhodes KJ, Monaghan MM, Barrezueta NX, Nawoschik S, Bekele-Arcuri Z, Matos MF, Nakahira K, Schechter LE, Trimmer JS (1996) Voltage-gated $\mathrm{K}^{+}$channel $\beta$ subunits: expression and distribution of $\operatorname{Kv} \beta 1$ and $\operatorname{Kv} \beta 2$ in adult rat brain. J Neurosci 16:4846-4860.

Saper CB, Sawchenko PE (2003) Magic peptides, magic antibodies: guidelines for appropriate controls for immunohistochemistry. J Comp Neurol 465:161-163.

Weiser M, Vega-Saenz de Miera E, Kentros C, Moreno H, Franzen L, Hillman D, Baker H, Rudy B (1994) Differential expression of Shaw-related $\mathrm{K}^{+}$channels in the rat CNS. J Neurosci 14:949-972. 